\title{
Increased Mercury release due to exposure to electromagnetic radiation as a limiting factor for using dental amalgam
}

\section{Paknahad', A. Dehghani Nazhvani ${ }^{2}$, S. Jarideh 3 , H. Mozdarani4, G. Mortazavi5 ${ }^{*}$, M. Haghani ${ }^{3}$, J. Eslami', S.M.J. Mortazavi' ${ }^{1,7}$}

\author{
${ }^{1}$ Oral and Dental Disease Research Center, Department of Oral and Maxillofacial Radiology, School of Dentistry, \\ Shiraz University of Medical Sciences, Shiraz, Iran \\ ${ }^{2}$ Biomaterial Research Center, Department of Oral and Maxillofacial Pathology, School of Dentistry, Shiraz \\ University of Medical Sciences, Shiraz, Iran \\ ${ }_{3}^{3}$ Ionizing and Non-ionizing Radiation Protection Research Centre (INIRPRC), Shiraz University of Medical \\ Sciences, Shiraz, Iran \\ ${ }^{4}$ Department of Medical Genetics, School of Medical Sciences, Tarbiat Modares University, Tehran, Iran \\ ${ }^{5}$ Dentist, Shiraz University of Medical Sciences, Shiraz, Iran \\ ${ }^{6}$ Anesthesiology Department, School of Nursing and Midwifery, Shiraz University of Medical Sciences, Shiraz, Iran \\ ${ }^{7}$ Department of Medical Physics, School of Medicine, Shiraz University of Medical Sciences, Shiraz, Iran
}

\section{- Short Report}

*Corresponding author:

Ghazal Mortazavi, DDS

Fax: +987112289113

E-mail:

mortazaviqaz@gmail.com

Revised: Jan. 2016

Accepted: Feb. 2016

Int. J. Radiat. Res., October 2016; 14(4): 355-359

DOI: 10.18869 /acadpub.jirr.14.4.355

\begin{abstract}
Background: Although it is one of the most toxic nonradioactive elements, mercury is widely used in dental amalgam. Mercury is a toxic element which can damage various organs such as central nervous system, renal, respiratory and hematologic systems. The adverse health impacts associated to exposure to some common sources of electromagnetic fields including laptop computers, mobile phones, MRI and mobile phone jammers have been evaluated by our laboratory in our previous investigations. In this study, we aimed to evaluate the effect of $X$ ray exposure on microleakage of amalgam restoration. Materials and Methods: Standardized class V cavities were prepared on the buccal surfaces of 46 non-carious freshly extracted human premolars. The teeth were randomly divided into experimental and control groups. Experimental group were exposed to $\mathrm{X}$-ray using an intraoral radiography machine at $60 \mathrm{kVp}, 0.1 \mathrm{~s}, 7 \mathrm{~mA}$ with $2.5 \mathrm{~mm}$ Al total filtration. The absorbed dose was $245.0 \pm 0.5 \mu \mathrm{Gy}$. All specimens were placed in $2 \%$ basic fuchsin solution for 24 hours. Then the specimens were sectioned and microleakage was assessed according to dye penetration using a stereomicroscope. Statistical analysis was performed with the Mann-Whitney U-test. Results: Microleakage was significantly higher in the X-ray exposed teeth compared to those of the non-irradiated samples. Conclusion: The results of the present study suggest that $\mathrm{X}$-ray exposure increased microleakage of amalgam restorations.
\end{abstract}

Keywords: X-ray, radiography, radiation, amalgam, microleakage, mercury.

\section{INTRODUCTION}

Dental amalgam is the predominant tooth filling material in posterior teeth restorations because of its high strength, low cost, durability and ease of manipulation $(1,2)$. However, lack of chemical adhesion to the tooth structure is one of the amalgam disadvantages which can result in pulp irritation, postoperative pain, secondary carries and tooth discoloration. Therefore, it is important to control marginal microleakage of amalgam restorations (3-5). Dental amalgam 
microleakage is defined as penetration of fluids, ions and bacteria to an interfacial gap between the cavity walls and the restorations (6). Microleakage has been suggested to be a significant problem leading to tooth discoloration around the margin of restoration, pulp pathology and secondary carries (7).

Increased release of mercury from dental amalgam fillings after exposure to MRI or microwave radiation emitted by mobile phones has been previously reported by our research team $(8,9)$. Moreover, our recent studies on the effects of stronger magnetic fields entirely confirmed our previous findings (10). From the other point of view, we have also shown that papers which reported no increased release of mercury after MRI, may have some methodological flaws (11). On the other hand, other investigators and our team have previously evaluated the impact of MRI on microleakage of amalgam restorations (12-15). Over the past several years, our laboratories have expanded their focus on studying the health effects of exposure to some common and/ or occupational sources of electromagnetic fields (EMFs) such as cellular phones, mobile base stations, mobile phone jammers, laptop computers, radars, dentistry cavitron(16). To the best of our knowledge, this is the first study that evaluates the effect of intraoral radiographies on amalgam microleakage. Therefore, this study aimed to investigate the microleakage of amalgam following common exposures to X-ray in intraoral radiographies.

\section{MATERIALS AND METHODS}

\section{Teeth samples}

This study was approved by the Ethics Committee of Shiraz University of Medical Sciences. Forty six non-carious premolar and molar teeth that were extracted for different reasons were used in this study. The teeth were stored in isotonic saline solution for not longer than 3 months after surface debridement. A standardized class V cavity was prepared on the buccal surface at the cementoenamel junction using carbide burs (SS White Burs, Lakewood,
NJ) and a high speed turbine under water spray. The cavity dimensions were $3 \mathrm{~mm}$ long occlusogingivally, $2 \mathrm{~mm}$ deep and $5 \mathrm{~mm}$ wide mesiodistally using a template. The cavities were restored with Cinalux (nongama2, spherical amalgam, Faghihi Dental, Tehran, Iran) amalgam. The amalgams were triturated according to manufacturers' directions, and then they were condensed incrementally towards the cavity walls using small condensers. All the procedures for restoration of the cavities including cavity preparation, burnishing, and polishing were performed by the same clinician. The restored teeth were stored in saline solution at $37^{\circ} \mathrm{C}$ for seven days. The teeth were randomly divided into two groups each containing 23 teeth.

\section{Irradiation of the samples}

A total of 23 teeth with amalgam-filled cavities were exposed to X-ray using a common intraoral radiography machine (Kodak 2100, Intraoral X-ray systems, France) at $60 \mathrm{kVp}, 0.1$ $\mathrm{s}, 7 \mathrm{~mA}$ with $2.5 \mathrm{~mm}$ Al total filtration $(1.5 \mathrm{~mm}$ of inherent and $1.0 \mathrm{~mm}$ of additional filtration) and a $20 \mathrm{~cm}$ film-target distance. Before irradiation, teeth samples were poured into a $500-\mathrm{mL}$ plastic tube. As discussed by Kursun et al. the thickness of the artificial saliva over teeth samples was $1.5 \mathrm{~cm}$ to mimic soft tissue (11). We used X-ray films (Fuji SHB films) for measuring the area of the exposure field $\left(4.7 \times 3.8 \mathrm{~cm}^{2}\right)$. Each sample was exposed 10 times to reduce variations. Dose measurement was performed using a Solid State Dosimeter (Solidose 400, Elimpex- Medizintechnik, Austria). The absorbed dose of the teeth samples was $245.0 \pm$ $0.5 \mu \mathrm{Gy}$.

\section{Microleakage evaluation}

The entire teeth surfaces were covered with two-layer application of nail varnish except for the restoration and $1 \mathrm{~mm}$ around the restoration margins. The samples were immersed in $2 \%$ basic fuchsin dye solution (Merck, Germany) for 24 hours and then were sectioned buccolingually with a slow speed water cooled saw. The section corresponding to the central portion of the tooth restoration was examined at the gingival, axial and occlusal margins under a 
stereomicroscope (Olympus. Tokyo, Japan) at 80X magnification by the examiner who was blinded to the groups.

The extent of microleakage was recorded according to the following $0-3$ scale criteria;

0 . No dye penetration

1. Dye penetration along the enamel

2. Dye penetration along the dentine-enamel junction (DEJ) but not including the axial wall

3. Dye penetration along the axial wall

The Mann-Whitney U-test was used to compare microleakage in the exposure and control groups to identify any statistically significant differences. $P$ values less than 0.05 were considered significant.

\section{RESULTS}

The distribution of the scores of microleakage in each group is summarized in table 1 . The scores of microleakage was significantly different in the exposure and control groups $(\mathrm{P}=0.03)$. The largest difference in the scale of microleakage between the exposed and control groups was observed for grade 0 and 2 microleakage. Interestingly, the proportion of the teeth with dye penetration along the dentine-enamel junction but not including the axial wall (grade 2) in exposure group was $21.7 \%$ while this proportion in the control group was only $4.3 \%$. Furthermore, the percent of the teeth with grade 3 in the exposed group was $8.7 \%$, while this percent was zero in the control group. Therefore, totally the rate of microleakage in the teeth exposed to X-ray in intraoral radiographies was nearly 6 times more than that of the control group (figures 1 and 2).

\section{DISCUSSION}

In this study it was revealed that the microleakage was significantly higher in the x-ray exposed teeth compared to those of the non-irradiated samples. These findings are generally in line with the results obtained in our previous studies on the increased release of mercury from dental amalgam fillings after exposure to different sources of electromagnetic fields such as MRI or radiations emitted by mobile phones $(8,10)$. To the best of our

Table 1. The distribution of the scores in the case and control groups

\begin{tabular}{|c|c|c|c|c|}
\hline \multirow{2}{*}{ Group } & \multicolumn{2}{|c|}{ Percentage of the scores (\%) } & \\
\cline { 2 - 5 } & 0 & 1 & 2 & 3 \\
\hline Control group & 52.6 & 26.3 & 10.5 & 10.5 \\
\hline $\mathrm{X}$ ray exposure group & 56.5 & 13 & 21.7 & 8.7 \\
\hline
\end{tabular}

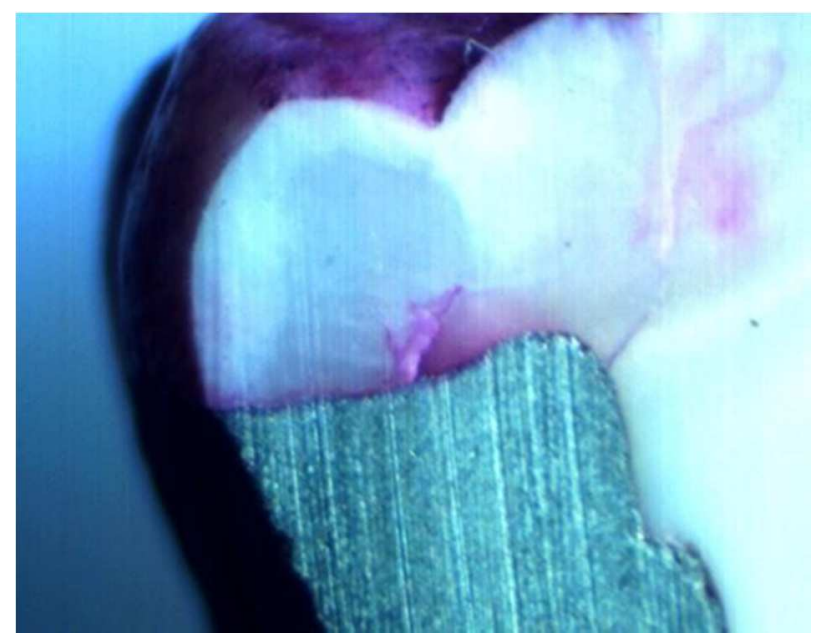

Figure 1. Dye peneteration through enamel, passing DEJ to dentin (score 3 ) in a X-rayed tooth.

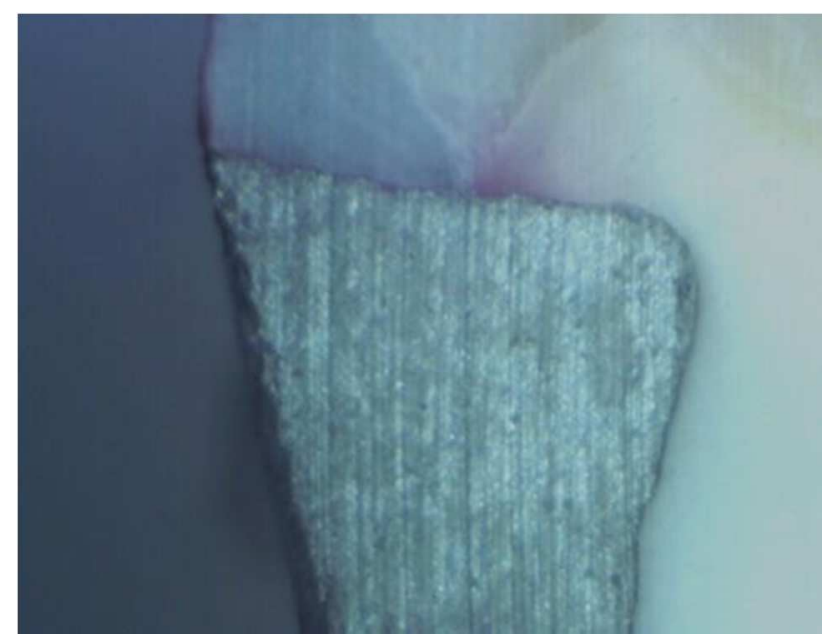

Figure 2. No Dye leakage (score 0 ) in a control tooth.

Int. J. Radiat. Res., Vol. 14 No. 4, October 2016 
knowledge, our study is the first to investigate the effects of mobile phone radiations on the release of mercury from dental amalgam fillings.

Our findings are in contrast with previously reported in vitro results of Müller-Miny etal. who examined the mercury release for typical MRI conditions, separately for static and variable magnetic fields, in a $1.5 \mathrm{~T}$ MR equipment (17). Muller-Miny and his colleagues could not show any significant increase in mercury release after MRI. We believe that the disagreement between our results and those reported by Müller-Miny may be due to the in vitro nature of their experiment. On the other hand, our observations are in line with those reported by Kursum et al. who measured the mercury release from amalgam restorations after X- ray exposures and showed that mercury release increases after exposure to X-ray (11). Mortazavi et al. have recently shown that a few published papers which reported no increased release of mercury after MRI, may have some methodological errors (18). They have also reported that increased mercury release after exposure to electromagnetic fields may be risky for the hypersensitive proportion of the population and pregnant women $(19,20)$.

Furthermore, there are only a few studies which examined the effect of MRI on the microleakage of amalgam restorations (12-14). While the results of two out of three of these studies suggest that MRI is not a completely safe technique in patients with amalgam restorations, Okgun et al. concluded that MRI does not increase microleakage of amalgam restorations. We believe that the increase in microleakage after exposure to X-ray in the present study may be due to high energy of the $\mathrm{x}$ -ray photons which may enable them to make chemical changes in the structure of amalgam. These chemical changes can be observed as alterations in chemical metallic bonds which may lead to gap formation.

Different methods have been used for evaluation of dental amalgam restorations microleakage. However, dye penetration test is a simple, inexpensive and qualitative method. However, as mentioned by Shahidi et al. more accurate techniques such as measuring the penetration of ammoniac silver nitrate between the tooth structure and amalgam could be more precise (12). Therefore, similar to the previous studies, we have used common visual microleakage scale for evaluation of microleakage. It is worth mentioning that a blot-like dye penetration along dentinal tubules was observed in this study which was similar to the pattern observed by Shahidi et al. in our previous study (12). However, Yilmaz and Misirlioğlu have observed a linear dye penetration pattern (14). Therefore, we used this technique for assessment of microleakage.

Methylene blue is cheap and better penetrative than eosin and other radioisotope traces. Therefore, methylene blue was used in the present study for dye penetration test.

In the present study, two layers of varnish coating were applied around restoration margins and the pulpal surfaces as a barrier to decrease microleakage around amalgam restoration and prevent undesirable dye penetration because varnishes did not bond to the tooth structure or amalgam restoration and they only perform as mechanical barriers. However, other adhesive liners such as resin based liners can decrease the microleakage of amalgam restorations and affect the results of the study.

In conclusion, the present study found high levels of microleakage in amalgam restorations exposed to X-ray compared to those of unexposed controls. Therefore, X-ray exposures may threaten the durability of amalgam restorations. Considering the significant importance of this challenging issue, further investigations are required to fully identify different aspects of the effect of exposure to electromagnetic fields on the microleakage and release of mercury from dental amalgam restorations.

\section{ACKNOWLEDGMENTS}

The authors thank the Vice-Chancellory of Research Shiraz University of Medical Science for supporting this research. The authors also thank Dr. Vosoughi of the Dental Research Development 
Center, of the School of Dentistry for the statistical analysis.

\section{Conflict of interest: Declared none.}

\section{REFERENCES}

1. Mitchell RJ, Koike M, Okabe T (2007) Posterior amalgam restorations--usage, regulation, and longevity. Dent Clin North Am, 51: 573-89.

2. Opdam N, Bronkhorst E, Loomans B, Huysmans M-C (2010) 12-year survival of composite vs. amalgam restorations. J dental research, 89: 1063-7.

3. Toledano M, Osorio E, Osorio R, Garcla-Godoy F (2000) Microleakage and SEM interfacial micromorphology of amalgam restorations using three adhesive systems. $J$ dent, 28: 423-8.

4. Berry F and Tjan A (1994) Microleakage of amalgam restorations lined with dentin adhesives. Am J Dent, 7: 333 -4 .

5. Cenci MS, Piva E, Potrich F, Formolo E, Demarco FF, Powers JM (2004) Microleakage in bonded amalgam restorations using different adhesive materials. Braz Dent J, 15: 13-8.

6. Kidd EA (1976) Microleakage: a review. J dent, 4: 199-206.

7. Morrow LA, Wilson NH, Setcos JC, Watts DC (2002) Microleakage of amalgam cavity treatment systems: an in vitro evaluation. Am J Dent, 15: 262-7.

8. Mortazavi S, Daiee E, Yazdi A, Khiabani K, Kavousi A, Vazirinejad R, et al. (2008) Mercury release from dental amalgam restorations after magnetic resonance imaging and following mobile phone use. Pakistan Journal of Biological Sciences, 11: 1142-6.

9. Paknahad M, Mortazavi S, Shahidi S, Mortazavi G, Haghani $M$ (2016) Effect of radiofrequency radiation from Wi-Fi devices on mercury release from amalgam restorations. Journal of Environmental Health Science and Engineering, 14: $12-6$.
10. Mortazavi SM, Neghab M, Anoosheh SM, Bahaeddini N, Mortazavi G, Neghab P, et al. (2014) High-field MRI and mercury release from dental amalgam fillings. Int J Occup Environ Med, 5: 101-5.

11. Kursun S, Öztas B, Atas H, Tastekin M (2014) Effects of Xrays and magnetic resonance imaging on mercury release from dental amalgam into artificial saliva. Oral Radiol, 30: 142-6.

12. Shahidi S, Bronoosh P, Alavi A, Zamiri B, Sadeghi A, Bagheri $M$, et al. (2009) Effect of magnetic resonance imaging on microleakage of amalgam restorations: an in vitro study. Dentomaxillofac Radiol, 38: 470-4.

13. Akgun OM, GuvenPolat G, Turanlllca A, Yildirim C, Demir P, Basak $F(2014)$ Does magnetic resonance imaging affect the microleakage of amalgam restorations? Iranian J Radiol, 11: e15565.

14. Yilmaz S and Misirlioğlu M (2013) The effect of 3 T MRI on microleakage of amalgam restorations. Dentomaxillofac Radiol, 42: 72-5.

15. Paknahad M, Shahidi S, Mortazavi SMJ, Mortazavi G, Moghadam MS, Nazhvani AD (2016) The Effect of Pulsed Electromagnetic Fields on Microleakage of Amalgam Restorations: An in Vitro Study. Shiraz E-Medical Journal, 17: e32329.

16. Mortazavi S, Parsanezhad M, Kazempour M, Ghahramani P, Mortazavi A, Davari M (2013) Male reproduc ve health under threat: Short term exposure to radiofrequency radia ons emi ed by common mobile jammers. J Hum Reprod Sci, 6: 124-8.

17. Müller-Miny H, Erber D, Möller H, Müller-Miny B, Bongartz $G$ (1996) Is there a hazard to health by mercury exposure from amalgam due to MRI? J Mag Res Imag, 6: 258-60.

18. Mortazavi SMJ and Mortazavi G (2014) Effects of X-rays and magnetic resonance imaging on mercury release from dental amalgam into artificial saliva. Oral Radiol, 1-2.

19. Mortazavi G and Mortazavi SMJ (2015) Amalgam contact hypersensitivity lesion: An unusual presentation-report of a rare case. Ann Med Health Sci Res, 5: 152-3.

20. Mortazavi G and Mortazavi SMJ (2014) Exposure to Electromagnetic Fields as a Hazard for People with Dental Amalgam Restorations. International Journal of Advances in Science and Technology, 153-5. 
